\title{
Childhood Lymphomatoid Granulomatosis
}

National Cancer Institute

\section{Source}

National Cancer Institute. Childhood Lymphomatoid Granulomatosis. NCI Thesaurus.

Code C123392.

Lymphomatoid granulomatosis that occurs during childhood. 